\title{
ALL OPERATORS ON A HILBERT SPACE ARE BOUNDED
}

\author{
BY J. D. MAITLAND WRIGHT \\ Communicated by Dorothy Stone, April 13, 1973
}

Introduction. Following Solovay [2], let ' $\mathrm{ZF}$ ' denote the axiomatic set theory of Zermelo-Fraenkel and let ' $\mathrm{ZF}+\mathrm{DC}$ ' denote the system obtained by adjoining a weakened form of the axiom of choice, DC, (see p. 52 of [2] for a formal statement of DC). From DC a 'countable' form of the axiom of choice is obtainable. More precisely, if $\left\{\boldsymbol{B}_{n}: n \in \boldsymbol{N}\right\}$ is a countable collection of nonempty sets then it follows from DC that there exists a function $f$ with domain $N$ such that $f(n) \in B_{n}$ for each $n$.

The system $\mathrm{ZF}+\mathrm{DC}$ is important because all the positive results of elementary measure theory and most of the basic results of elementary functional analysis, except for the Hahn-Banach theorem and other such consequences of the axiom of choice, are provable in $\mathrm{ZF}+\mathrm{DC}$. In particular, the Baire category theorem for complete metric spaces and the closed graph theorem for operators between Fréchet spaces are provable in $\mathrm{ZF}+\mathrm{DC}$.

Solovay shows [2] that the proposition, Each subset of the real numbers is Lebesgue measurable, cannot be disproved in $\mathrm{ZF}+\mathrm{DC}$. He does this by constructing a model for $\mathrm{ZF}+\mathrm{DC}$ in which the proposition becomes a true statement.

We shall see that the proposition, Each linear operator on a Hilbert space is a bounded linear operator, is consistent with the axioms of $\mathrm{ZF}+\mathrm{DC}$. Other results of this type are obtained. For example, Whenever $X$ and $Y$ are separable Fréchet groups and $h: X \rightarrow Y$ is a homomorphism then $h$ is continuous, cannot be proved or disproved in $\mathrm{ZF}+\mathrm{DC}$.

Fortunately all the hard work in model theory has been done by Solovay. All that we use here is straightforward functional analysis.

All operators on a Hilbert space are bounded. We recall that a subset $S$ of a topological space $T$ is said to have the Baire property if there exists an open set $U$ such that $(U \backslash S) \cup(S \backslash U)$ is meagre. Let BP be the proposition: Each subset of a complete separable metric space has the Baire property. In [2, $\$ 4]$, Solovay outlines an argument which shows that when BP is interpreted in his model for $\mathrm{ZF}+\mathrm{DC}$ then it becomes a true statement. Hence BP is consistent with the axioms of $\mathrm{ZF}+\mathrm{DC}$ provided Solovay's model exists. We adjoin BP as an axiom and denote the extended system by ' $\mathrm{ZF}+\mathrm{DC}+\mathrm{BP}$ '.

AMS (MOS) subject classifications (1970). Primary 47A99, 02K05; Secondary 46B99, $46 \mathrm{C} 10$. 
In this paper certain propositions will be shown to be theorems of $\mathrm{ZF}+\mathrm{DC}+\mathrm{BP}$. It is easy to show, by a Hamel base argument, that for each such proposition its negation is a theorem in ZFC (ZF with the axiom of choice adjoined). So these propositions can neither be proved nor disproved in ZF + DC, provided Solovay's model exists.

Let I be the axiom: There exists an inaccessible cardinal. Solovay uses the hypothesis that there exists a (transitive) model for $\mathrm{ZFC}+\mathrm{I}$ when constructing his model.

From now onward we work in $\mathrm{ZF}+\mathrm{DC}+\mathrm{BP}$. All our theorems are derived in this system.

LEMMA 1. Let $X$ and $Y$ be separable metric spaces and let $X$ be complete. Let $f: X \rightarrow Y$ be any function mapping $X$ into $Y$. Then there exists a meagre set $N \subset X$ such that the restriction of $f$ to $X \backslash N$ is continuous.

Choose $\varepsilon>0$. Let $\left\{y_{r}: r=1,2, \ldots\right\}$ be a countable dense subset of $Y$. For each $r$, let $S_{r}$ be the open sphere centred on $y_{r}$ with radius $\varepsilon / 2$. Then $Y=\bigcup_{1}^{\infty} S_{r}$.

Let $A_{1}=S_{1}$ and, for $n \geqq 1$, let $A_{n+1}=\left(\bigcup_{1}^{n+1} S_{r}\right)-\left(\bigcup_{1}^{n} S_{r}\right)$. So $Y=\bigcup_{1}^{\infty} A_{n}$, where each $A_{n}$ is contained in an open sphere of radius $\varepsilon / 2$ and $A_{i} \cap A_{j}=\varnothing$ for $i \neq j$.

Let $B_{n}=f^{-1}\left[A_{n}\right]$ for $n=1,2, \ldots$ Then $X=\bigcup_{1}^{\infty} B_{n}$ and $B_{i} \cap B_{j}=$ $\varnothing$ for $i \neq j$.

For any $n, B_{n}$ has the Baire property and so there is an open set $U_{n}$ and a meagre set $M_{n}$, where $M_{n}=\left(B_{n} \backslash U_{n}\right) \cup\left(U_{n} \backslash B_{n}\right)$, such that $U_{n} \cap\left(X \backslash M_{n}\right)=B_{n} \cap\left(X \backslash M_{n}\right)$. Let $M$ be the meagre set $\bigcup_{1}^{\infty} M_{n}$. Then $U_{n} \cap(X \backslash M)=B_{n} \cap(X \backslash M)$ for each $n$. Thus $B_{n} \cap(X \backslash M)$ is an open subset of $X \backslash M$ in the relative topology of $X \backslash M$.

Let $J$ be the set of all natural numbers $n$ for which $B_{n} \cap(X \backslash M)$ is not empty. By DC there exists a function $\xi$ with domain $J$ such that $\xi(n) \in B_{n} \cap(X \backslash M)$ for each $n$. Let $h$ be the function defined on $X \backslash M$ by $h(x)=f(\xi(n))$ whenever $x \in B_{n} \cap(X \backslash M)$.

Let $\left(z_{j}\right)(j=1,2, \ldots)$ be any sequence in $X \backslash M$ which converges to a point $z$ in $X \backslash M$. Then, for some $n \in J, B_{n} \cap(X \backslash M)$ is an open neighbourhood of $z$ in the relative topology of $X \backslash M$. So there exists a natural number $k$ such that $z_{j} \in B_{n} \cap(X \backslash M)$ whenever $j \geqq k$. Thus $h\left(z_{j}\right)=h(z)$ whenever $j \geqq k$. So $h:(X \backslash M) \rightarrow Y$ is continuous. Whenever $x \in X \backslash M$ then $x \in B_{n} \cap(X \backslash M)$ for some $n \in J$ and thus

$$
d(h(x), f(x))=d(f(\xi(n)), f(x))<\varepsilon .
$$

By putting $\varepsilon=1 / m(m=1,2, \ldots)$ we can find a sequence of functions $\left(h_{m}\right)(m=1,2, \ldots)$ and a sequence of meagre sets $\left(N_{m}\right)(m=1,2, \ldots)$ such that $h_{m}$ is a continuous map of $X \backslash N_{m}$ into $Y$ and $d\left(h_{m}(x), f(x)\right)<1 / m$ 
for each $x \in X \backslash N_{m}$. Let $N$ be the meagre set $\bigcup_{1}^{\infty} N_{m}$. Then $\left(h_{m}\right)(m=$ $1,2, \ldots$ ) converges uniformly to $f$ on $X \backslash N$. So $f$ is continuous on $X \backslash N$.

THEOREM 2. Let $X$ and $Y$ be separable metrizable topological groups and let $X$ be complete. Let $H: X \rightarrow Y$ be any group homomorphism. Then $H$ is continuous.

Let $\left(x_{n}\right)(n=1,2, \ldots)$ be a sequence in $X$ converging to a point $x$. By Lemma 1 , there is a meagre set $M$ such that $H$ is continuous when restricted to $X \backslash M$.

By the Baire category theorem, which is valid in $\mathrm{ZF}+\mathrm{DC}$, there exists $z \in X$ such that $z$ is not in the meagre set $x^{-1} M \cup \bigcup_{1}^{\infty}\left(x_{n}^{-1} M\right)$. Thus $x z \in X \backslash M$ and $x_{n} z \in X \backslash M$ for each $n$. Hence $H(x z)=\lim H\left(x_{n} z\right)$. Since $H$ is a homomorphism, $H(z)=\lim H\left(x_{n}\right)$.

The elegant argument used in Theorem 2 is due to Banach, see Theorem 4, Chapter 1 [1]. I wish to thank Professor A. Wilansky for drawing my attention to this reference.

In the following we do not require Fréchet spaces to be locally convex.

THEOREM 3. Let $X$ be any Fréchet space and let $Y$ be a separable metrizable topological vector space. Let $T: X \rightarrow Y$ be a linear map. Then $T$ is continuous.

Let $\left(x_{n}\right)(n=1,2, \ldots)$ be any sequence in $X$ which converges to zero. Let $X_{0}$ be the closed linear span of $\left\{x_{n}: n=1,2, \ldots\right\}$ so that $X_{0}$ is a separable Fréchet space. Then, by the preceding theorem, the restriction of $T$ to $X_{0}$ is continuous. Thus $T x_{n} \rightarrow 0$ as $n \rightarrow \infty$. So $T$ is continuous.

COROLlary 4. Each linear functional on a Fréchet space is continuous.

Theorem 5. Let $X$ and $Y$ be Fréchet spaces and let $T: X \rightarrow Y$ be a linear map. If there exist enough functionals on $Y$ to separate the points of $Y$ then $T$ is continuous.

Let $\left(x_{n}\right)(n=1,2, \ldots)$ be a sequence in $X$ converging to $x$ and suppose $\left(T x_{n}\right)(n=1,2, \ldots)$ converges to $y$. For any functional $\phi$ on $Y, \phi$ is continuous on $Y$ and $\phi T$ is continuous on $X$. Thus

$$
\phi(y)=\lim \phi\left(T x_{n}\right)=\lim \phi T\left(x_{n}\right)=\phi T(x) .
$$

So $T x=y$. It now follows by the closed graph theorem that $T$ is continuous.

It must be emphasised that discontinuous linear operators, defined on incomplete spaces, arise naturally in ZF + DC. For example, there is an abundance of unbounded operators defined on dense subspaces of a Hilbert space. But, for linear operators defined on the whole of a Hilbert space the following theorem holds in $\mathrm{ZF}+\mathrm{DC}+\mathrm{BP}$. 
THEOREM 6. Let $H$ be a Hilbert space and let $T: H \rightarrow H$ be a linear operator defined on the whole of $H$. Then $T$ is bounded.

Let $H$ be any Hilbert space. Then, for each nonzero $x$ in $H$, the linear functional $f$, defined by $f(y)=\langle y, x\rangle$, does not vanish at $x$. So $H$ has a separating family of linear functionals.

This implies that, in ZFC, we cannot obtain discontinuous operators on (the whole of) a Hilbert space except by invoking an 'uncountable' form of the axiom of choice.

\section{REFERENCES}

1. S. Banach, Théorie des opérations linéaires, Monografje Mat., PWN, Warsaw, 1932.

2. R. M. Solovay, A model of set theory in which every set of reals is Lebesgue measurable, Ann. of Math. (2) 92 (1970), 1-56. MR 42 \#64.

Department of Mathematics, University of Reading, Reading, England 